\title{
Patterns pedagógicos: possibilidades para a docência com as tecnologias
}

\section{Pedagogical patterns: possibilities for teaching with technologies}

\author{
SIMONE MARKENSON \\ Universidade Estácio de Sá
}

\section{GISELLE MARTINS DOS SANTOS FERREIRA}

Pontifícia Universidade Católica do Rio de Janeiro

\begin{abstract}
Resumo: O desafio de integrar tecnologias às práticas pedagógicas tem se tornando mais premente à medida que novos recursos se tornam disponíveis em intervalos cada vez menores. A partir de uma revisão bibliográfica, identificamos propostas para disseminação das práticas pedagógicas com as tecnologias, a partir da teoria de patterns. Observou-se, contudo, que se trata de uma visão reducionista em comparação com a proposta original. Este artigo apresenta um recorte de pesquisa que analisou as concepções de patterns na Educação, revelando pouco consenso em relação às formas de identificação, catalogação, compartilhamento e, efetivamente, aplicação no contexto da docência com tecnologias educacionais. Adicionalmente, pouca empiria sobre o impacto da abordagem de patterns nas práticas pedagógicas foi encontrada. Comparando com a abordagem aplicada à computação, com conceitos e práticas bem estabelecidas, percebese a necessidade de adequação da teoria às diferentes propostas e atores na construção de práticas pedagógicas com as tecnologias.
\end{abstract}

Palavras-chave: Patterns pedagógicos. Docência com as TIC. Tecnologia educacional.

\begin{abstract}
The challenge of integrating technologies into pedagogical practices has become more and more pressing as new features become available at ever smaller intervals. From a literature review, we identified proposals for the dissemination of pedagogical practices with technologies, from the theory of patterns. However, it was observed that this is a reductionist view compared to the original proposal. This paper presents a research study carried out to analyze the conceptions of patterns in education, revealing little consensus regarding the forms of identification, cataloging, sharing and, effectively, application in the context of teaching with educational technologies. Additionally, little empiric evidence on the impact of pattern approach on pedagogical practice was found. Compared with the approach applied to computing, with wellestablished concepts and practices, one perceives the need to adapt the theory to the different proposals and actors in the construction of pedagogical practices with technologies.
\end{abstract}

Keywords: Pedagogical patterns. Teaching with ICT. Educational Technology 


\section{Introdução}

O desafio de ensinar com as tecnologias da informação e comunicação (TIC) não é novo, mas foi ampliado com as possibilidades de uso de uma grande quantidade de artefatos digitais e da infraestrutura de comunicação propiciada pela Internet. Espera-se que o docente esteja preparado para tirar proveito das TIC sem que o uso seja impositivo, em uma esfera administrativa, pois a imposição de uma mudança em suas práticas para algo diferente do que Ihe é familiar não favorecerá a apropriação necessária para aproveitamento de novos recursos. A crescente oferta de produtos em intervalos curtos reduz o tempo disponível para abstração e reflexão sobre a vocação de uso de cada um, e, portanto, a incorporação das experiências docentes com tecnologias às práticas pedagógicas.

Coll, Mauri e Onrubia (2010) observaram que docentes e estudantes, em diferentes medidas e mesmo instrumentalizados, não se "relocalizavam" e, assim, mantinham os padrões de ensinoaprendizagem das gerações anteriores. A pesquisa feita em 2017 pelo Centre for Learning \& Performance Technologies (HART, 2017) mostrou que as ferramentas que são mais utilizadas nos processos educacionais continuam sendo as de "produtividade", tais como editores de texto, planilhas e aplicativos para elaboração de apresentação, além de sites de busca e compartilhamento de documentos e vídeos. No relatório de 2008, as primeiras posições foram ocupadas por ferramentas de classificação de informações (indexadores) para buscas na internet e navegadores, revelando pouca inovação nesses quase dez anos e reforçando o uso das TIC em papéis coadjuvantes em vez de mediadoras no processo de ensino-aprendizagem.

A adoção de abordagens pedagógicas com as TIC depende tanto da posição epistemológica adotada quanto do enfrentamento dos mitos que rondam o universo da tecnologia educacional. Selwyn (2014, p. viii) descreveu a falta de questionamento sobre o uso da tecnologia educacional como "uma alucinação consensual por parte de educadores tecnoromânticos", embora admita que alguns educadores possam ser movidos por um interesse genuíno em uma educação de qualidade. A dificuldade em se estabelecer uma relação causal entre as práticas com as TIC e a melhoria da aprendizagem, bem como a falta de compreensão sobre a medida em que as TIC modificam as práticas pedagógicas (COLL; MAURI; ONRUBIA, 2010) evidenciam que a discussão sobre o tema ainda é necessária.

A abordagem de patterns busca estabelecer conexões entre a experimentação e a prática pedagógica, organizadas para concretizar expectativas educacionais específicas (FRANCO, 2016) com as TIC. Originalmente, essa abordagem foi utilizada como um conjunto de parâmetros para projetos de arquitetura considerando não somente parâmetros técnicos, como as medidas, mas principalmente o aspecto humanizado refletido pela observação da utilização real de cada espaço. Posteriormente foi adotada, com modificações importantes, pela ciência da computação e, desde o final dos anos 1990, é objeto de pesquisa na Educação, em especial na tecnologia educacional, como uma forma de consolidar práticas pedagógicas com as TIC e "incorporar a reflexão contínua e coletiva, de forma a assegurar que a intencionalidade proposta é disponibilizada a todos" (FRANCO, 2016). 
Este artigo é um recorte de uma pesquisa de doutorado cujo objetivo foi analisar as concepções e práticas com patterns na área da Educação. Em particular, este artigo focaliza as possibilidades dos patterns como apoio à integração de TIC nas práticas de ensino-aprendizagem. Na seção 2 a metodologia utilizada é apresentada. A seção 3 apresenta a proposta original de Alexander (1979) e a seção 4 uma revisão de literatura no âmbito da Educação. Por fim, na seção 5 , discutiremos os resultados obtidos.

\section{Metodologia}

A abordagem metodológica utilizada foi a da pesquisa qualitativa e bibliográfica, buscando a origem do processo de "transposição" da teoria, da arquitetura para a educação, desde a primeira publicação sobre patterns na educação, conhecidos como patterns pedagógicos.

Para a pesquisa de tese, foram identificadas 394 publicações, entre artigos, anais de congresso, teses e livros, partindo de busca em bases de dados acadêmicas disponibilizadas pela internet, abertas ou não, como mostra sinteticamente o Quadro 2, utilizando os termos "pedagogical pattern*" ou "learning pattern*" ou "teaching pattern*" ou "educational pattern*" ou "padr* pedagógico*", associados à "educação" ou "education" ou "pedagogia" ou "pedagogy" e ainda ao nome do autor de referência, "Alexander".

Quadro 2 - Número de publicações identificadas inicialmente

\begin{tabular}{|l|l|}
\hline \multicolumn{1}{|c|}{ Base } & \multicolumn{1}{|c|}{ Resultado } \\
\hline Banco de Teses (Capes) & 32 \\
\hline Science@Direct & 74 \\
\hline ACM & 164 \\
\hline Open Research Online & 17 \\
\hline Google Scholar & 107 \\
\hline TOTAL & 394 \\
\hline
\end{tabular}

Fonte: Elaborada pelos autores.

Após a seleção de um conjunto representativo de publicações, utilizamos um encadeamento de referências para identificarmos os textos seminais e excluir as repetições ou variações sobre um mesmo tema. Foram excluídas referências relacionadas diretamente à computação, não aderentes ao objeto da pesquisa ou repetidas em diferentes bases. Selecionamos um conjunto de 50 publicações para entendermos de que formas os patterns pedagógicos podem contribuir para a discussão das práticas pedagógicas com as TIC. Para que as referências selecionadas pudessem ser confrontadas com as propriedades dos patterns, tal qual proposto por Alexander (1979), foi necessário conhecer mais detalhadamente como a abordagem foi construída e como foi incorporada por outras áreas. 


\title{
3 A origem dos patterns
}

Em 1977, o arquiteto austríaco Christopher Alexander publicou a obra A Pattern Language: Towns, Buildings, Construction com o objetivo de promover o desenvolvimento de projetos de arquitetura, principalmente para não-arquitetos. Um conjunto de "boas práticas" que poderiam ser combinadas para resolver diferentes escalas de projetos, desde cidades até pequenos detalhes em uma casa. A teoria que subsidiou essa obra só foi publicada dois anos depois, com o título The Timeless Way of Building, esclarecendo que a metodologia proposta não se restringia à transmissão de experiências e descrevendo a ideia de projetos em termos de patterns, definindo o termo:

\begin{abstract}
Cada padrão (sic) descreve um problema que ocorre repetidas vezes em nosso meio ambiente e então descreve o ponto central da solução do problema, de modo que você possa usar a mesma solução milhares de vezes, mas sem jamais ter de repeti-la (ALEXANDER; ISHIKAWA; SILVERSTEIN, 2013, p. xiv).
\end{abstract}

Cabe observar que foi utilizado o termo "padrão" por ser uma citação da obra já traduzida para o português. A definição de padrões industriais refere-se à palavra standard; porém, o termo pattern, não tem uma tradução mais adequada para o português além de, novamente, a palavra "padrão". Manteremos o termo em inglês para não gerar dúvidas sobre o que está em discussão, por não se tratar de um conjunto de especificações para um determinado propósito, como definido pela International Organization for Standardization (ISO).

A abordagem de patterns foi construída em um processo de amadurecimento sobre a visão de projetos de arquitetura, iniciado quando Alexander morou na Índia, em 1961, em uma vila de cabanas de barro com apenas uma construção de tijolos que lembrava um templo. Geração após geração, novas construções eram realizadas, nos mesmos moldes das anteriores, sem que houvesse qualquer registro que indicasse como e porque construir daquela forma. As construções eram perfeitamente adaptadas ao contexto local, atendiam aos requisitos de conforto e segurança da população, e não contavam com o apoio de arquitetos. Por não conseguir expressar as características dessas construções em uma única palavra, de uma forma poética, denominou esse conjunto de características em uma "qualidade sem nome", conceito esse que permeia toda a obra. Leitner (2015) identificou que essa é uma limitação da língua inglesa e que, em alemão, o adjetivo lebendig representaria bem esse conjunto. Reconheceu, no entanto, que a utilização do acrônimo "QWAN" (quality without a name) trazia um toque místico à obra de Alexander. Seria algo equivalente, mas não exato, à qualidade de ser vívido e que promovesse um sentimento de unidade.

As "boas construções", na visão de Alexander, apresentavam um núcleo invariante representado pelo instinto humano de buscar o pertencimento. Observou que as construções onde as pessoas se sentiam confortáveis haviam sido feitas com a participação de quem estava no centro do processo; eram construções vivas. A necessidade de municiar essas pessoas, não obrigatoriamente arquitetos, de parâmetros e ferramentas que as permitisse criar projetos únicos foi o ponto de partida para o desenvolvimento dos patterns na arquitetura, descrito funcionalmente: 
[...] na forma de uma regra que estabelece uma relação entre um contexto, um sistema de forças que emergem do contexto e uma configuração que permite que as forças se resolvam nesse contexto (ALEXANDER, 1979, p. 253, tradução nossa).

O "contexto" define as características do ambiente onde o problema está inserido; as "forças" são os elementos que levariam à direção contrária do que se pretende obter e a configuração é a solução do problema no contexto especificado, considerando as forças existentes. Alexander (1979) citou como exemplo a experiência de uma casa dinamarquesa antiga com uma grande sala com recantos destacados abertos para a parte comum da sala de tal forma que pessoas nos diferentes espaços poderiam interagir, mesmo fazendo coisas diferentes. Essa solução resolvia um sistema de forças particular, em que temos uma sala suficientemente grande, habitada por pessoas que possuem atividades ou hobbies que exigem um espaço para que possam ser exercidas, mas que gostam de estar juntas na maior parte do tempo. Por questões de organização e segurança, a área comum não é utilizada para a prática dos hobbies individuais. Sinteticamente, o sistema de forças a ser resolvido é definido pelo conflito entre privacidade e convivência em um contexto de uma sala compartilhada, cuja solução foi a utilização de recantos conectados por uma parte comum. A solução foi nomeada como Nichos em um catálogo como 253 patterns construído a partir de experiências, testes e observações em diferentes condições, ao longo de aproximadamente 10 anos (ALEXANDER; ISHIKAWA; SILVERSTEIN, 2013). A escolha de um conjunto de patterns para solução de um determinado problema constitui uma linguagem de patterns. A observação de atividades ou situações que ocorrem repetidamente em um espaço físico é a chave para o entendimento de projetos com patterns, e obtê-los requer tempo e disponibilidade para validação.

Os patterns capturam a experiência de especialistas para comunicá-las a quem precisar e resolver problemas recorrentes equilibrando contextos e forças em conjunto com outros patterns. São estruturas que relacionam componentes de um sistema em uma relação de equilíbrio, gerando regras simples para sistemas complexos. É importante reforçar que os patterns são parâmetros de projetos, cuja função não se limita à reutilização simples de soluções já executadas. Cada solução é única e não pode ser obtida apenas pela identificação em um catálogo, porque catálogos podem gerar um sistema de forças sem solução. A posição de uma poltrona perto da janela com vista para um muro, por exemplo, expressa a dicotomia entre querer ficar confortavelmente acomodado em um poltrona e ser repelido pela mesma poltrona, porque seu posicionamento não propicia uma experiência agradável. É uma contradição interna e deve ser evitada.

Dez anos depois da publicação de A Pattern Language: Towns, Buildings, Construction, pesquisadores oriundos da Computação buscaram na teoria proposta por Alexander (1979) um referencial para compartilhamento e disseminação de "boas práticas" no desenvolvimento de sistemas de computador. No entanto, parte do arcabouço conceitual foi deixado de lado, e somente as questões relativas ao compartilhamento de conhecimento foram mantidas na abordagem da computação. A reutilização de código, aumentando o grau de abstração no desenvolvimento de sistemas, também pode ser comparada, ainda que de forma rudimentar, aos aspectos que motivaram o desenvolvimento dos patterns na arquitetura. A ideia de utilizar 
soluções conhecidas para um determinado problema é bastante atrativa, principalmente quando implica redução de tempo e de esforço no desenvolvimento de um projeto.

Uma das primeiros propostas de aplicação de patterns no desenvolvimento de sistemas foi registrada por Beck e Cunninghan (1987), por terem tido dificuldade para terminar a interface de um sistema. Para resolverem o problema, inseriram no processo quem iria utilizar o sistema, seguindo as orientações fornecidas por Alexander (1979), oferecendo ferramental adequado para viabilizar a discussão. Apresentaram um pequeno conjunto de cinco patterns, que foram utilizados para a construção da interface de janelas desejada. Não se tratava ainda de uma linguagem de patterns, mas indicava uma preocupação com aspectos parecidos com os de Alexander em relação à arquitetura. Em 1994, Beck e Cunningham se uniram a Ralph Johnson, Grady Boock e Jim Coplien e fundaram o The Hillside Group, com o propósito de fortalecer a relação entre os patterns de Alexander e a programação, em especial utilizando-se o paradigma da orientação a objetos.

Um pouco depois da proposta inicial de Beck e Cunningham (1987), Gamma et al (1993) publicaram o artigo que, posteriormente, se tornaria referência dos patterns na computação. Os quatro autores (Erich Gamma, Richard Helm, Ralph Johnson, John Vlissides) também são conhecidos como "Gang of Four" (GoF). O termo GoF é utilizado como referência aos patterns desenvolvidos por eles, apresentados como uma abordagem para reutilização de soluções de software. Utilizaram um vocabulário comum para designar estruturas que se comportariam como blocos de estruturas reutilizáveis na construção de sistemas complexos. Utilizaram a denominação design patterns para diferenciá-los da linguagem de patterns, deixando explícito que o conjunto apresentado não formava uma linguagem:

\footnotetext{
Design patterns capturam soluções que se desenvolveram ao longo do tempo. Portanto, eles não são o projeto que as pessoas tendem a gerar inicialmente. Eles refletem incontáveis "reprojetos" e "reprogramações", já que os desenvolvedores têm lutado para uma maior reutilização e flexibilidade em seus programas (GAMMA et al., 1994, p. xi, tradução nossa).
}

Apesar da inspiração nos trabalhos de Alexander (1979), a proposta dos autores capturava apenas uma parte da essência original, voltada para a transmissão de conhecimento adquirido para desenvolvedores de software e reutilização de soluções de programação para problemas recorrentes. No âmbito da computação, patterns são estratégias utilizadas para definir e desenvolver estruturas de software. Ao contrário do que prega Alexander (1979), na computação os autores sugerem que somente se utilize design patterns quando a flexibilidade for realmente necessária, uma vez que são inseridos no desenvolvimento de sistemas níveis intermediários adicionais, que podem tornar o projeto mais complexo. A apropriação pela computação se efetivou com a publicação do livro de Gamma et al. (1994), em que foram apresentados 23 patterns. Os patterns, na concepção do GoF, se adequaram tão bem aos propósitos da computação que há quem pense ser essa sua área de origem, e não a Arquitetura.

Seguindo a influência da arquitetura e da computação, os projetos utilizando patterns também se desenvolveram na Educação, assumindo-se que "uma linguagem de patterns pedagógicos pode ser um caminho para capturar soluções fundamentais para os problemas diários de ensino" 
(FINCHER, 1999b, p. 8, tradução nossa). Em uma das primeiras publicações identificadas sobre o uso pedagógico da concepção de patterns, Fincher (1999b) ressaltou que o termo pattern possui uma única origem, referindo-se à obra de Alexander, mas adverte para a confusão que o uso de uma palavra tão comum pode gerar quando representa um novo conceito.

\section{Patterns Pedagógicos}

Na área da Educação, a propagação das tecnologias da informação e comunicação (TIC) impulsionou a adoção da abordagem de patterns, a partir do final dos anos 1990, principalmente pelos pesquisadores oriundos da computação, e atuantes também na Educação, incluindo Fincher (1999b), Goodyear (2004), Conole, McAndrew e Dimitriadis (2011), Bergin et al (2012), Mor et al (2014), Köppe (2015) e Fassbinder, Barbosa e Magoulas (2017). Embora a metodologia não seja específica para as práticas pedagógicas com as TIC, o recorte se faz necessário considerando as especificidades da transição das práticas docentes com as tecnologias para um universo reflexivo, crítico e com intencionalidade clara. As tecnologias apresentam, quase sempre, vocação para uma determinado propósito, porém "nas práticas pedagogicamente construídas, há a mediação do humano e não a submissão do humano a um artefato técnico previamente construído" (FRANCO, 2016).

Considerando apenas os aspectos de registro e comunicação de práticas, não parece fazer uma grande diferença das outras formas já consolidadas. Em especial no contexto educacional, os planos de aula e os padrões estabelecidos do learning design já cumpririam esse papel. A diferença está no fato de patterns não serem implementações. Descrevem "quando, como e por que" alguma coisa deve ser criada, mas não cria. Analogamente, na arquitetura não há diretrizes sobre quantidades e materiais para uma construção. Também na computação, não são programas em uma linguagem de programação específica e sim orientações estruturais de como escrever o programa.

A influência de pesquisadores oriundos da Ciência da Computação, em função da experiência no tema, se evidenciou com o Pedagogical Patterns Project (PPP), iniciado em 1996, como uma ideia apresentada em um congresso anual sobre programação, sistemas, linguagens e aplicações orientadas a objetos (OOPSLA'95). Na época, havia uma concentração de docentes envolvidos nos processos de ensino do paradigma da programação orientada a objetos, na época muito controverso (SHARP; MANNS; ECKSTEIN, 2003). Havia o mito de que seria necessário esquecer os paradigmas de desenvolvimento de sistemas anteriores para que fosse possível aprender o novo e, nesse contexto, ter uma forma de reutilizar e compartilhar experiência pareceu ser uma boa proposição. A teoria de patterns já era conhecida no meio para uma funcionalidade semelhante no desenvolvimento de sistemas e o termo pattern foi utilizado também como uma forma de capturar experiências bem-sucedidas nos processos de ensino-aprendizagem da orientação a objetos.

Os idealizadores do PPP, Mary Lynn Manns, Phil McLaughlin, Maximo Prieto e Helen Sharp, iniciaram o registro de experiências de outros professores durante o congresso europeu de programação orientada a objetos (ECOOP'96) e em outros congressos durante o ano de 1997. 
No final de 1997, já haviam sido coletados 50 candidatos a patterns propostos por autores de diferentes países. Essa diversidade fomentou estruturas com focos bem diferentes e que não conversavam facilmente entre si, mas, segundo os autores, isso não era um problema naquele momento. No congresso de 2000 (OOPSLA 2000) conduziram oficinas direcionadas a domínios mais restritos na área e as relações entre esses domínios. Foram produzidas quatro linguagens de patterns nos dois anos subsequentes. As conferências anuais, PLoP e EuroPLoP, delimitavam o prazo para produção de patterns e linguagens para que pudessem ser revisadas por outros autores. Percebe-se nitidamente essa preocupação quando verificamos a sequência de trabalhos publicados. Em alguns casos, parte da linguagem é publicada em um congresso e a outra parte em outro, como feito por Köppe (2011a) e (2011b). Depois de algumas mudanças de formato, o grupo optou pelo formato utilizado por Alexander (1979) em função do suporte à relação entre patterns e por não ter detalhes excessivos que os deixem datados.

A partir dos resultados do PPP, foram produzidas cinco linguagens, publicadas posteriormente em livro (BERGIN et al., 2012): aprendizagem ativa, feedback, aprendizagem experiencial, ganhando diferentes perspectivas, ensinando a partir de diferentes perspectivas. Cada conjunto possui um primeiro pattern, que funciona como uma chave de acesso aos demais em um total de 85 itens coletados e catalogados. Para ilustrar a linguagem desenvolvida, o pattern "diferentes níveis de exercícios" é um dos componentes de "tornar o estudante ativo", proposto originalmente por Fricke e Völter (2000), sugerindo a prática de uma habilidade recém-adquirida através de exercícios com diferentes níveis de dificuldade, de diferentes tópicos e abordagens. Os autores admitiram, no entanto, que os patterns produzidos pelo PPP não seguiam exatamente a proposta de Alexander (1979), porém entendiam que era uma primeira abordagem ao tema.

Fincher (1999a) deu início à generalização da abordagem para aspectos além do ensino da programação. Em uma sequência de publicações, analisou elementos comuns nos trabalhos de Alexander (1979), Gamma et al. (1994) e do PPP para identificar possíveis elementos comuns e necessários para caracterizar um pattern e uma linguagem de patterns. Inicialmente, pretendia verificar a possibilidade de desenvolver uma linguagem para o estudo de interfaces humanocomputador, porém não se restringiu a esse aspecto. De uma forma geral, os patterns pedagógicos foram descritos de modo a serem utilizados na sua concepção mais elementar: a comunicação de práticas. A característica de que "patterns não são criados, são garimpados nas boas práticas" (BERGIN, 2013, p. 7, tradução nossa) é um ponto de contato com a teoria de Alexander (1979) e segue um formato geral, composto por uma descrição do problema e uma proposta não prescritiva de solução.

A obtenção de um conjunto de patterns passa por um processo de identificação de candidatos a partir da observação das práticas. O processo é denominado "mineração", em alusão ao termo utilizado no universo da computação na área Banco de Dados (data mining). Posteriormente, o conjunto obtido é catalogado construindo-se, assim, uma linguagem para resolver um problema específico. Porém a observação não é suficiente para garantir que estamos falando de uma prática pedagógica. 
Winters e Mor (2008) sugeriram uma metodologia para desenvolvimento participativo de projetos interdisciplinares tecno-pedagógicos com o objetivo de cumprir a etapa de identificação de candidatos. A metodologia, identificada como IDR (identificação-desenvolvimentorefinamento), é baseada nos processos de elicitação de requisitos, técnica também oriunda da computação para a etapa de levantamento, utilizando-se entrevistas com todos os envolvidos no processo. No contexto, as entrevistas têm a função de provocar o "surgimento" dos patterns. A elicitação de patterns não é uma ação trivial. É preciso identificar partes "invariantes" que possam ser generalizadas. Para tal, Winters e Mor (2008) propuseram ferramentas para que os participantes pudessem descrever suas práticas e para que essas descrições pudessem ser comparadas, com o intuito de identificação das partes comuns e, como consequência, das generalizações. Posteriormente, Mor, Warburton e Winters (2012) ampliaram a metodologia IDR utilizando uma estrutura denominada Participatory Patterns Workshops (PPW), composta por um conjunto de oficinas nas quais as experiências dos professores eram descritas e o conhecimento incorporado nas narrativas organizado sob a forma de patterns, que, por sua vez, foram identificados como soluções para possíveis cenários.

Como exemplo, tomaremos a linguagem Seminar (FRICKE; VÖLTER, 2000) composta por 48 patterns e indicada por Bergin (2000) como a melhor linguagem de patterns pedagógicos apresentada, pela compatibilidade com a proposta original de Alexander (1979). Acrescenta-se ao mérito da linguagem, a intencionalidade e validação esperada de uma prática pedagógica. A linguagem é utilizada para descrever as fases de preparação, execução e avaliação de um seminário, ilustrada na Figura 1. Cada fase é composta por um conjunto de patterns, iniciando e terminando com alguma forma de validação.

Figura 1 - Estrutura da Linguagem Seminars

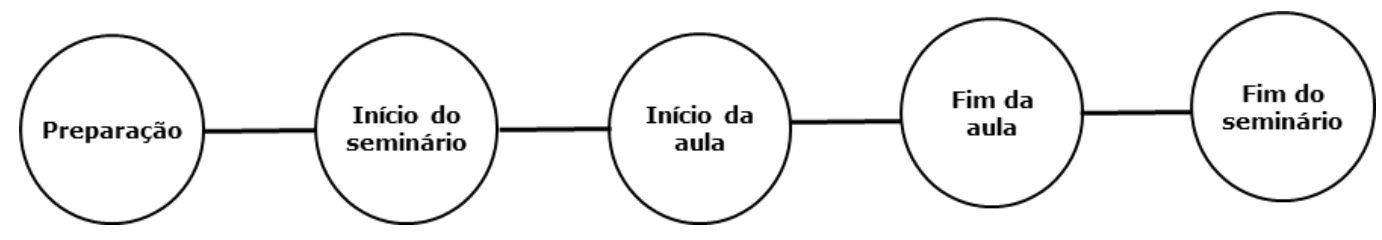

Fonte: Elaborada pelos autores (FRICKE; VÖLTER, 2000)

A fase de preparação estrutura o conteúdo e cria um ambiente confortável para os participantes. O início do seminário é marcado por um ritual acolhedor de boas-vindas aos participantes e o início da aula estabelece o começo da apresentação do conteúdo. A fase que representa o fim da aula estabelece um conjunto de possibilidades para validação final do aprendizado e término do conteúdo. Essa validação, no entanto, não é restrita a uma atividade final, pois entre início e fim da aula existe um conjunto de atividades. Por fim, a última etapa se refere ao fim do seminário como o momento de considerações e avaliação. Um recorte da linguagem que aborda a etapa de preparação é apresentado na Figura 2. 
Figura 2 - Recorte da Linguagem Seminars - fase de preparação

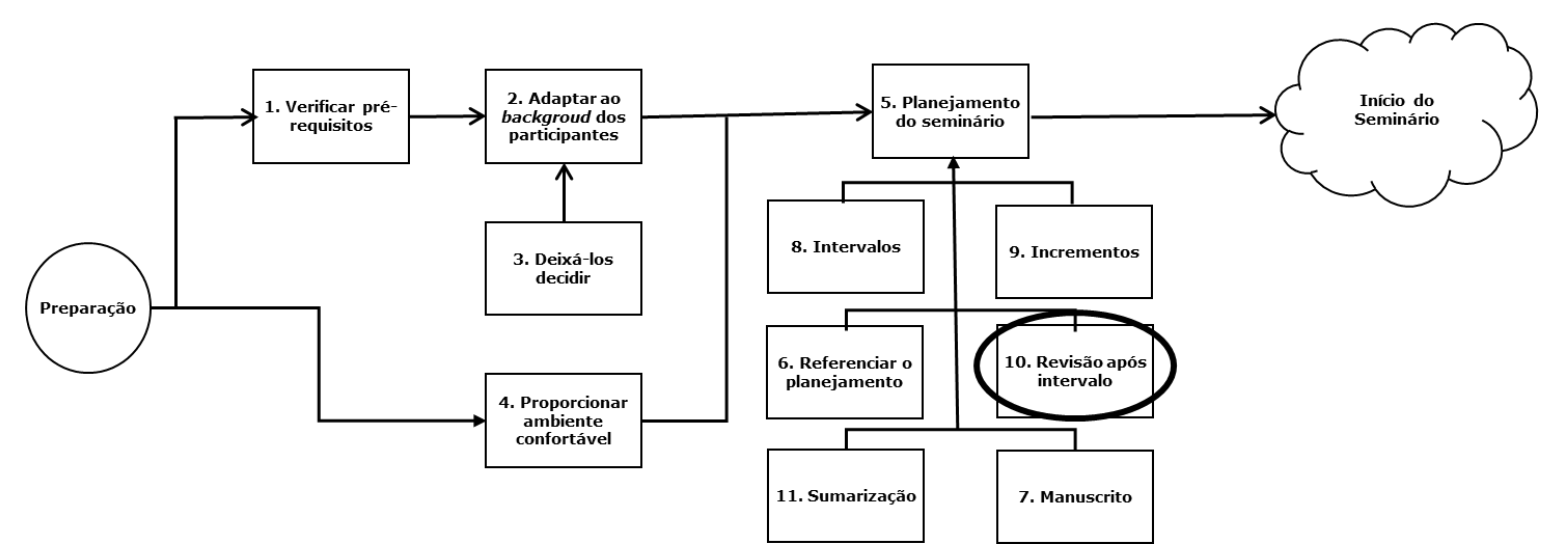

Fonte: Adaptado de Fricke e Völter (2000)

O exemplo do Quadro 1 apresenta a tradução do pattern REVISÃo APós INTERVALO, que propõe uma atividade de revisão após um intervalo em um seminário ou em uma aula expositiva. Estão presentes os elementos fundamentais para ser considerado um pattern: problema, contexto, forças e solução. A solução enfatiza o que deve ser feito e não como deve ser feito, demonstrando a função não prescritiva dos patterns.

Quadro 1 - Pattern REVISÃO APÓS INTERVALO

REVISÃO APÓS INTERVALO **

Intervalos servem como um caminho para relaxar e ter Personal Communication. No entanto, os participantes tendem a esquecer o que foi ensinado antes do intervalo, porque os tópicos ainda não foram armazenados na memória de longo prazo. Adicionalmente, você precisa dar chance aos participantes de fazer conexões com o tópico anterior ao intervalo e o novo tópico.

$$
\text { * * }
$$

Portanto, após cada intervalo, revise brevemente os tópicos abordados antes da interrupção. Concentre-se nos aspectos importantes, tente não se perder nos detalhes. Leve em conta esse tempo adicional quando você criar a agenda e o PLANEJAMENTO do SEMINÁRIO.

Existem diferentes maneiras de realizar esta revisão. Ou você simplesmente repete os tópicos importantes, ou você revisa rapidamente as transparências importantes etc. Em um grupo bem trabalhado, é ainda melhor se um dos participantes repetir os tópicos importantes, talvez até usando suas transparências.

Como sempre, é melhor se os participantes estiverem ativos em vez de passivos. Aqui, isso significa que a repetição pode ser alcançada ao permitir que cada participante anote os tópicos importantes em um papel por conta própria, ou discutindo os temas importantes com seu vizinho. Para simplificar essa discussão, você pode preparar cartões com tópicos importantes. Outra possibilidade é fazer perguntas e deixar os participantes respondê-las, talvez em grupos.

Fonte: (FRICKE; VÖLTER, 2000, p. 14, tradução nossa)

Uma linguagem construída com o rigor necessário poderia ser aplicada na construção de uma prática pedagógica e assim disponibilizada a todos. Curiosamente, após extensa busca de referências, identificamos apenas quatro relatos de validação de linguagens em campo, sendo apenas dois específicos para práticas com as TIC. Como proposta de uso geral, Bauer e Baumgartner (2010) desenvolveram e aplicaram uma dinâmica de apresentação de trabalhos 
pelos alunos. Vale ressaltar que a prática foi proposta e aplicada pelos autores, o que não garante a validação necessária. A segunda referência é uma tese que estabelece uma relação dos patterns estilos de aprendizagem centrado no ensino técnico profissionalizante (AMARAL, 2015). Em relação a apropriação das TIC, mesmo com aderência parcial a teoria de Alexander (1979), a temática gira em torno da ciência da computação, como, por exemplo, no ensino de desenvolvimento de interfaces humano-computador (DEARDEN et al., 2002) e de recursos educacionais abertos (CONOLE; MCANDREW; DIMITRIADIS, 2011).

De acordo com a teoria proposta por Alexander (1979), a obtenção de um pattern se faz por observação da atuação de um "especialista" com a intenção de descrever a essência de um problema e sua respectiva solução para uma audiência maior e não obrigatoriamente especializada. Em contextos como o de e-learning, considerando a definição de e-learning como "o uso de tecnologias digitais da informação e da comunicação como suporte e apoio em processos de ensino-aprendizagem, tanto na formação a distância quanto presencial" (ADELL; BELLVER; BELLVER, 2010, p. 245), a especialização vem de profissionais de diferentes áreas, tais como da psicologia, ciência da computação e educação, cada qual com a sua perspectiva sobre o tema.

O caráter multidisciplinar da área traz alguns fatores adicionais ao já tão complexo processo de mineração. Além disso, a identificação do que é ou não um pattern nem sempre é trivial, e pode haver confusão com uma prática docente que já tenha funcionado bem em uma situação específica. É importante reforçar que um pattern carrega uma intenção de abstração de uma prática testada e avaliada repetidas vezes. A "regra dos três" é particularmente aplicada por grupos originários da computação, para validação de um pattern somente após três instâncias de uso (FINCHER, 1999b). Essa abordagem é difícil de ser adotada na educação, pois a observação do que ocorre em uma sala de aula nem sempre é possível.

Nos últimos 10 anos, a pesquisa em patterns pedagógicos deu origem a concepções distintas para a inserção das TIC nos processos educacionais. Entre os pioneiros na elaboração de patterns para atividades acadêmicas, Anthony (1996) e Bergin (2000), ambos originários da área de Computação, apresentaram uma coletânea com 14 patterns, cada um, voltados para os cursos de Ciência da Computação. Anthony (1996), no entanto, generalizou a finalidade dos patterns para o ensino de tópicos técnicos considerados difíceis pelo autor como, por exemplo, a programação orientada a objetos. Bergin foi mais específico, mantendo o foco nos cursos de Computação, mas admitindo que alguns deles poderiam ser utilizados em outras áreas. Além da estrutura, Anthony (1996) e Bergin (2000) têm em comum o direcionamento para o público profissional, mesmo que iniciante.

Mor et al (2014) organizaram uma coletânea de patterns, de diferentes autores, voltados para o uso de tecnologia e categorizados em quatro grandes grupos: Projetos centrados no aluno; Comunidades de aprendizagem; Mídias sociais e interação do aluno em espaços sociais; Avaliação e feedback. Para cada grupo, são fornecidos um conjunto de patterns, as narrativas que originaram os patterns e cenários em que serão aplicados, formando assim uma linguagem para o tema. Os patterns foram obtidos em oficinas e, em vez de serem prescritivos, forneciam 
"soluções abertas para necessidades concretas" (MOR et al., 2014, p. 2, tradução nossa). Köppe et al.(2015) propuseram uma linguagem composta por cinco patterns, direcionada à sala de aula invertida, para que os alunos estivessem bem preparados para a aula presencial, exigindo uma mudança de atitude do docente. O conhecimento prévio é obtido pelos alunos, cada um em seu próprio tempo, por meio de vídeos, leituras complementares ou outras formas de obtenção de informação, como visita a museus, por exemplo. As aulas possuem um formato diferente da aula expositiva tradicional, pois parte do princípio de que as informações necessárias já são de conhecimento do aluno.

Os patterns apresentados neste artigo foram obtidos em oficinas pela experiência de docentes na prática de sala de aula invertida e na literatura da área. O formato adotado segue trabalhos semelhantes do mesmo autor (KÖPPE, 2011a). Abordagem semelhante foi utilizada no desenvolvimento de uma linguagem para cursos abertos, online e massivos (FASSBINDER; BARBOSA; MAGOULAS, 2017), conhecidos pela sigla MOOC, composta por 39 patterns organizados em oito categorias, exemplificada em um estudo de caso na área de ensino da engenharia de software. As duas concepções citadas diferenciam-se tanto pela forma de mineração, quanto pela estrutura da linguagem. No primeiro caso, são construídas narrativas coletivas para, posteriormente, identificar possíveis patterns. No segundo, há um cenário específico a ser tratado, como a adoção de sala de aula invertida, e as práticas observadas são direcionadas para um problema específico. Nesse caso, a proposta e a representação estão mais próximas da abordagem de Alexander (1979).

\section{Considerações finais}

Inicialmente, identificamos nas publicações selecionadas, seis categorias segundo os propósitos de pesquisa sobre patterns pedagógicos:1) teoria sobe patterns pedagógicos; 2) mineração e práticas para identificação e registro de patterns a partir da observação de práticas docentes, não limitada a contextos de uso da tecnologia;3) catalogação; 4) estudos empíricos de práticas com patterns fora do contexto das TIC; 5 ) estudos empíricos de práticas com patterns no contexto das TIC e 6) gerenciadores de catálogos. A participação de cada categoria na amostra selecionada está apresentada no Quadro 3.

Quadro 3 - Distribuição de publicações por categoria.

\begin{tabular}{|l|l|l|}
\hline \multicolumn{1}{|c|}{ Id. } & \multicolumn{1}{|c|}{ Descrição } & Participação \\
\hline 1 & Teoria: discutem aspectos teóricos dos patterns pedagógicos. & $38 \%$ \\
\hline 2 & $\begin{array}{l}\text { Mineração: enfatizam estratégias e práticas para identificação e registro de patterns a partir de } \\
\text { práticas docentes, não limitada a contextos de uso da tecnologia. }\end{array}$ & $11 \%$ \\
\hline 3 & Catalogação: tratam da organização dos patterns obtidos na etapa de mineração. & $36 \%$ \\
\hline 4 & Aplicação sem TIC: discutem práticas realizadas fora do contexto das TIC. & $4 \%$ \\
\hline 5 & Aplicação com TIC: discutem práticas realizadas no contexto das TIC. & $4 \%$ \\
\hline
\end{tabular}


Os números apresentados na caracterização da literatura nos mostram a existência de um movimento no sentido de desenvolvimento dos patterns pedagógicos. Constatamos também que $45 \%$ da literatura selecionada foi publicada em anais de congressos e que $54 \%$ se referem a abordagem aplicada ao e-learning, o que reforça a posição de desenvolvimento do processo de construção da teoria e elaboração de referenciais mais homogêneos voltados para a tecnologia. No entanto, fica clara a falta de publicações sobre práticas pedagógicas desenvolvidas com patterns, em especial enfatizando tecnologias. Identificamos nas publicações na área da Educação discussões que abordavam pattern como subsídio para transmissão de práticas de ensino, enfatizando o uso de tecnologias, porém sem a apropriação para que se tornassem práticas pedagógicas. Adicionalmente, a definição original de patterns (ALEXANDER, 1979) é repetida como um mantra, tanto por autores na área da Computação quanto da Educação, sem, no entanto, passar da visão reducionista da simples transmissão de conhecimento. Como já discutido, não é só essa a função dos patterns.

Um aspecto positivo identificado nos relatos das práticas de mineração é o grau de integração e comprometimento entre os participantes. O processo de construção torna-se mais efetivo que o produto; por si só, um fator de contribuição para reposicionamento dos docentes e disseminação de práticas pedagógicas, em especial com as TIC. A construção dos patterns pedagógicos atua na conciliação entre o docente e a tecnologia educacional, reduzindo o tempo de absorção de conhecimento por parte do docente e eliminando uma possível rejeição a práticas com as TIC por falta de domínio. As TIC são, por vezes, referenciadas como alavancas potenciais para novas práticas pedagógicas. No entanto, é necessário que haja apropriação e motivação por parte do docente.

Na Educação, o que se observa, é a concentração de autores e pesquisadores oriundos da área da Computação e que ainda trazem consigo os aspectos dos patterns adotados no desenvolvimento de sistemas. De uma certa forma, a fundamentação teórica encontrada em algumas publicações, quando existem, se restringem aos aspectos concretos, como a estrutura, o fato de serem obtidos das práticas e de resolverem problemas recorrentes. São aspectos relacionados ao que os patterns fazem e não o que eles são. Falta a essência. Falta a inspiração humanística, que não está presente nos patterns computacionais.

As produções relacionadas ao e-learning identificam, mesmo que parcialmente, as TIC como mediadora de atividades conjuntas para apoio os alunos e TIC como configuradores de ambientes ou espaços de trabalho e de aprendizagem virtuais para autoinstrução. As propostas apresentadas são distintas para contextos com as TIC e contextos sem as TIC, e ainda diferentes quanto à intenção de uso das TIC, o que não acontece nem na arquitetura, nem na computação. $\mathrm{Na}$ arquitetura, uma mesma linguagem é utilizada como referência independentemente do tipo de construção. Da mesma forma, na computação, o catálogo GoF é utilizado para desenvolvimento de qualquer tipo de sistema. Tanto na arquitetura quanto na computação há 
uma obra de referência, que ainda não foi idealizada na Educação. A falta de convergência entre as propostas de elaboração de patterns pedagógicos de diferentes autores, inclusive em relação a sua estrutura, dificulta uma comparação generalista com os aspectos da teoria de Alexander (1979), como foi feito com a computação.

Há registros de elaboração de oficinas e dos patterns obtidos nas discussões e trocas de experiências. No entanto, identificamos apenas dois relatos de aplicação das linguagens de patterns por outros que não os do próprio autor, impossibilitando garantir que os objetivos das oficinas de mineração, ou outras abordagens para levantamento de patterns, sejam atingidos e, portanto, se tornarem práticas pedagógicas. Os registros das práticas elaborados de forma estruturada são, no entanto, importantes para que, em algum momento, possam ser compartilhados, atendendo assim uma das premissas da teoria de patterns. Adicionalmente, ainda não há um convencimento de que os patterns produzidos sejam realmente capazes de gerar práticas melhores.

Existem lacunas para a efetiva utilização dos patterns nos processos de ensino-aprendizagem no que se refere a rotinas que possam realimentar e consolidar as linguagens existentes. 0 desenvolvimento de um catálogo de referência estabelecendo minimamente um conjunto testado de estratégias levaria as linguagens de patterns a cumprir seu papel na consolidação das práticas pedagógicas, em especial com as TIC. Acreditamos que sejam questões para estudos futuros e que tenhamos fornecido subsídios para que novas explorações sejam realizadas.

\section{Referências}

ADELL, J.; BELLVER, A. J.; BELLVER, C. Ambientes virtuais de aprendizagem e padrões de e-learning. In: COLL, C.; MONEREO, C. (Eds.) Psicologia da educação virtual: aprender e ensinar com as tecnologias da informação e da comunicação. Tradução de Naila Freitas. Porto Alegre: Artmed, 2010. Cap. 12, p. 245-267.

ALEXANDER, C. The timeless way of building. New York: Oxford University Press, 1979.

ALEXANDER, C.; ISHIKAWA, S.; SILVERSTEIN, M. Uma linguagem de padrões: a pattern language. Tradução de Alexandre Salvaterra. Porto Alegre: Bookman, 2013. 1171 p.

AMARAL, M. P. Uso de Padrões Pedagógicos em Consonância com Estilos de Aprendizagem: um levantamento centrado no Ensino Técnico Profissionalizante. 2015. 125f. Tese-Resumo(Doutorado em Ensino de Ciências e Matemática)- Universidade Cruzeiro do Sul, São Paulo. Disponivel em: <https://sucupira.capes.gov.br/sucupira/public/consultas/coleta/trabalhoConclusao/viewTrabalhoConclusao .jsf?popup=true\&id_trabalho=2887274>. Acesso em: 10 jan. 2018.

ANTHONY, D. L. G. Patterns for classroom education. In: VLISSIDES, J. M.; COPLIEN, J. O.; KERTH, N. L. (Eds.) Pattern languages of program design 2. Boston: Addison-Wesley, 1996. p. 391-406.

BAUER, R.; BAUMGARTNER, P. The Potential of Christopher Alexander's Theory and Practice of Wholeness: Clues for Developing an Educational Taxonomy. In: EuroPLoP '10, 2010. Anais... New York: ACM, 2010. p. 12.1-12.21.

BECK, K.; CUNNINGHAM, W. Using Pattern Languages for Object-Oriented Programs. In: OOPSLA'87 workshop on Specification and Design for Object-Oriented Programming, 1987, Orlando. Anais... New York: ACM, 1987.

BERGIN, J. Fouteen Pedagogical Patterns. In: European Conference on Pattern Languages of Programms (EuroPLoP '2000), 5. Anais... Irsee: [s.n.], 2000. Disponivel em: <http://www.coldewey.com/europlop2000/>. Acesso em: 12 jan. 2018. 
BERGIN, J. Patterns Courses $2000 . \quad$ Slides, Disponivel <http://csis.pace.edu/ bergin/patterns/PatternCourseSlides.pdf>. Acesso em: 15 jul. 2019.

BERGIN, J. Writing patterns: software, organizational, pedagogical. USA: Slant Flying Press, 2013.

BERGIN, J. et al. Pedagogical Patterns: Advice for Educators. [S.I.]: Joseph Bergin Software Tools, 2012.

COLL, C.; MAURI, T.; ONRUBIA, J. A incorporação das tecnologias da informação e comunicação na educação: do projeto técnico pedagógico às práticas de uso. In: COLL, C.; MONEREO, C. (Eds.) Psicologia da Educação Virtual: aprender e ensinar com as tecnologias da informação e da comunicação. Tradução de Naila Freitas. Porto Alegre: Artmed, 2010. Cap. 3, p. 66-93.

CONOLE, G.; MCANDREW, P.; DIMITRIADIS, Y. The role of CSCL pedagogical patterns as mediating artefacts for repurposing Open Educational Resources. In: POZZI, F.; PERSICO, D. (Eds.) Techniques for Fostering Collaboration in Online Learning. Hershey: IGI Global, 2011. p. 206-233.

DEARDEN, A. et al. Using Pattern Languages in Participatory Design. In: BINDER, T.; GREGORY, J.; WAGNER, I. (Eds.) Participatory Design Conference, 2002, Malmö, Sweden. Anais... Palo Alto: [s.n.], 2002. p. 104113. Disponivel em: <http://shura.shu.ac.uk/3/1/fulltext.pdf>. Acesso em: 10 nov. 2015.

FASSBINDER, A. G. D. O.; BARBOSA, E. F.; MAGOULAS, G. D. Developing an Educational Design Pattern Language for MOOCs. In: CREDINÉ SILVA DE MENEZES, J. M. (Ed.) Simpósio Brasileiro de Informática na Educação (SBIE 2017), 28, Recife. Anais... Porto Alegre: SBC, 2017. p. 456-465. Disponivel em: <http://www.br-ie.org/pub/index.php/sbie/article/view/7574>. Acesso em: 12 fev. 2018.

FINCHER, S. What is a Pattern Language? In: Late-Breaking Papers, Chi'99 Human Factors in Computing Systems. Anais... Pittsburgh, Pennsylvania: ACM, 1999a.

FINCHER, S. Analysis of Design: an exploration of patterns and pattern languages for pedagogy. Journal of Computers in Mathematics and Science Teaching: special issue CS-ED Research, v.18, n. 3, p.331-348, dez. 1999b.

FRANCO, M. A. D. R. S. Prática pedagógica e docência: um olhar a partir da epistemologia do conceito. Revista Brasileira de Estudos Pedagógicos, Brasília, v. 97, n. 247, p. 534-551. dez. 2016. Disponivel em: <ttp://www.scielo.br/scielo.php?script=sci_arttext\&pid=S2176-66812016000300534\&lng=en\&nrm=iso> . Acesso em: 15 jul. 2019.

FRICKE, A.; VÖLTER, M. SEMINARS: A Pedagogical Pattern Language about teaching seminars effectively. In: European Conference on Pattern Languages of Programs (EuroPLoP '2000), 5. Anais... Irsee: [s.n.], 2000. p. 87-128. Disponivel em: <http://www.coldewey.com/europlop2000/papers/voelter+fricke.zip>. Acesso em: 1 nov. 2017.

GAMMA, R. et al. Design Patterns: Abstraction and Reuse of Object-Oriented Design. In: NIERSTRASZ, O. (Ed.) European Conference on Object-Oriented Programming, VII, 1993, Kaiserslautern. Anais... London: Springer-Verlag, 1993. p. 406-431.

GAMMA, R. et al. Design Patterns: elements of reusable object-oriented software. Westford: AddisonWesley, 1994. 395 p.

GOODYEAR, P. Patterns, pattern languages and educational design. In: ATKINSON, R. et al. (Eds.) Beyond the comfort zone. ASCILITE Conference, 21st, 2004, Perth. Anais... [S.I.]: ASCILITE, 2004. p. 339-347. Disponivel em: <http://www.ascilite.org/conferences/perth04/procs/pdf/goodyear.pdf>. Acesso em: 12 set. 2014.

HART, J. C4LPT. Centre for Learning \& Performance Technologies, 2017. Disponivel em: <http://c4lpt.co.uk/>. Acesso em: 10 fev. 2018.

ISO. International Organization for Standization. Disponivel em: <http://www.iso.org/iso/home/standards.htm>. Acesso em: 9 jan. 2018.

KÖPPE, C. A Pattern Language for Teaching Design Patterns (Part 1). In: European Conference on Pattern Languages of Programs (EuroPLoP '11), 16., 2011, Irsee. Anais... New York: ACM, 2011a.

KÖPPE, C. A Pattern Language for Teaching Design Patterns (Part 2). In: Conference on Pattern Languages of Programs (PLoP) , 18., 2011, Portland. Anais... New York: ACM, $2011 \mathrm{~b}$. 
KÖPPE, C. Towards a Pattern Language for Lecture Design: An inventory and categorization of existing lecture-relevant patterns. In: European Conference on Pattern Languages of Programs (EuroPLoP) ,18, 2013, Irsee, Germany. Anais... New York: ACM, 2015.

KÖPPE, C. et al. Flipped Classroom Patterns: designing Valuable In-Class. In: European Conference on Pattern Languages of Programs (EuroPLoP '15), 20, 2015, Kaufbeuren. Anais... New York: ACM, 2015.

LEITNER, H. Pattern Theory: Introduction and prespectives on the tracks of Christopher Alexander. Graz: HLS, 2015.

MOR, Y. et al. Using design patterns to develop and share effective practice. In: MOR, Y. et al. (Eds.) Pratical Desing Patterns for Teaching and Learning with Technology. Rotterdan: Sense Publishers, v. 8, 2014. Cap. Introduction. (Trails in education: Technologies that support navigarional learning).

MOR, Y.; WARBURTON, S.; WINTERS, N. Participatory pattern workshops: a methodology for open learning design inquiry. Research in Learning Technology, v.20, n. 30, ago. 2012. Disponivel em: <http://www.researchinlearningtechnology.net/index.php/rlt/article/view/19197>. Acesso em: 10 abr. 2015.

SELWYN, N. Distrusting educational technology: critical questions for changing times. New York: Routledge, 2014.

SHARP, H.; MANNS, M. L.; ECKSTEIN, J. Evolving pedagogical patterns: the work of the Pedagogical Patterns Project. Computer Science Education, v.13, n. 4, p.315-330, 2003.

WINTERS, N.; MOR, Y. IDR: A participatory methodology for interdisciplinary design in technology enhanced learning. Computers \& Education, v.50, p.579-600, 2008.

Recebido em setembro de 2018.

Aprovado para publicação em agosto de 2019.

\section{Simone Markenson}

Universidade Estácio de Sá, - UNESA, Brasil,

simone@pech.com.br

\section{Giselle Martins dos Santos Ferreira}

Pontifícia Universidade Católica do Rio de Janeiro - PUC-RJ, Brasil, giselle-ferreira@puc-rio.br 